\title{
Cross-sectioning Sub-millimetre Sized Defects in Ornamental Chrome-plated Component
}

\author{
A.J. Lockley ${ }^{1}$ \\ ${ }^{1 .}$ Canadian Nuclear Laboratories, Chalk River Labs, Chalk River Ontario Canada
}

Ornamental chrome components are desirable due to their lustrous and smooth appearance; however blemishes can occur and undermine their appearance. Finding the root-cause of such blemishes is of great importance to manufacturers so that process changes or improvements can be made. In one such case an ornamental chrome component had developed rust-coloured stains after field testing. The component comprised a flash chrome plating on a nickel-plate base supported by a steel substrate. A unique combination of metallographic- and microscopymethods were used to isolate the source of the staining.

A digital microscope (DM) in conjunction with a Scanning Electron Microscope (SEM) was a synergistic tool set effective in locating and imaging small surface defects. The DM is a recent evolution of the optical microscope that was particularly useful for its ability to scan wide fields of view at lower magnification with the ability to zoom to higher magnification without having to change objective lenses while maintaining superior depth of field compared with standard optical methods. This instrument proved invaluable for the detection of small circular defects frequently associated with surface stains as seen in Figure 1. Circular defects were examined using the SEM to obtain higher resolution images as seen in Figure 2. The circular defects ranged in size from about $100 \mu \mathrm{m}$ to $200 \mu \mathrm{m}$ in diameter.

Cross-sectioning a feature between $100 \mu \mathrm{m}$ and $200 \mu \mathrm{m}$ is challenging using standard metallographic techniques as the removal rates of material of such a thickness can occur in seconds during grinding operations. This being the case serial sectioning by grinding can be a risky operation when features of evidence are rare. To increase the probability of successfully cross-sectioning one of these sub-millimetre defects, sections for examination were mounted in a transparent mounting material. Although the mounting material was transparent it was still difficult to detect small features inside the mount. To facilitate visual contact with the small features an optically clear "window" was made on the side of the mount by grinding and polishing a facet on the side of the mount parallel to the surface containing the defect. Serial sectioning by grinding was used but progression was monitored by observation into the mount with the aid of a stereo microscope.

Visually aided serial-sectioning was successful in producing metallographic cross-sections through the centre of the circular defects. This revealed that circular defects tended to be isolated to the outer surface of the nickel plating and if they occurred in a region where the nickel plate was sufficiently thick the defects had limited depth as seen in Figure 3. However, in regions where the nickel plate was relatively thin, a pit in the steel substrate was commonly observed as seen in Figure 4. The circular nature of the defects suggest they result from the presence of gas bubbles that prevented a uniform coverage of the surface. Corrosion to the steel substrate would occur in areas where nickel plating thickness was inadequate to provide corrosion protection. As corrosion progressed the corrosion by-products seeped out of the pit and stained the chrome surface. 


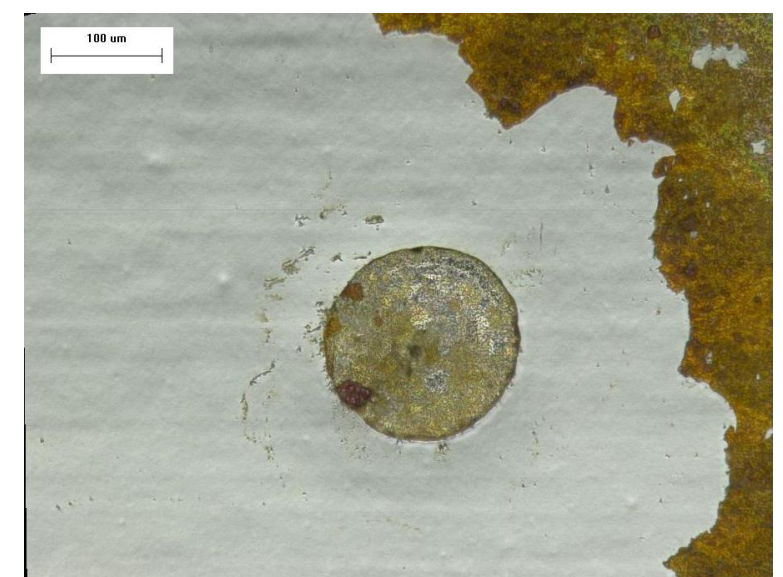

Figure 1 Digital Microscope image showing a circular defect with surrounding rust-coloured staining on a chrome plated surface.

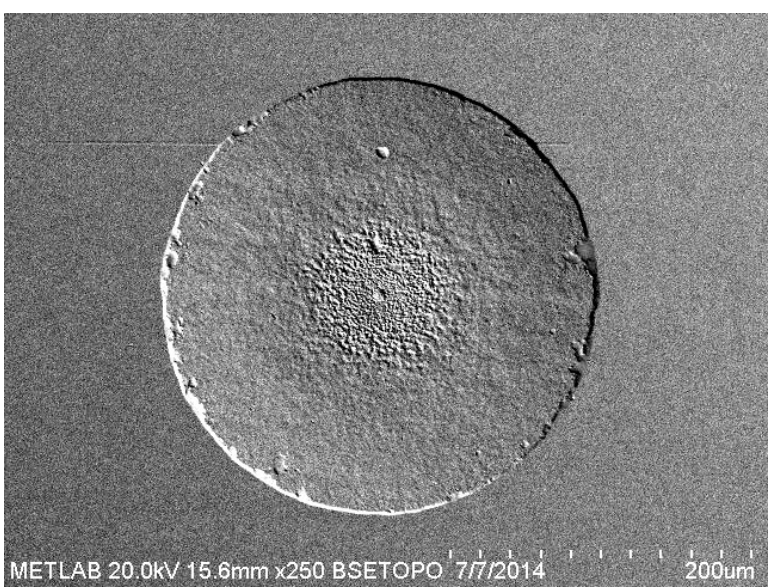

Figure 2 An SEM image showing detail of one of the circular defects.

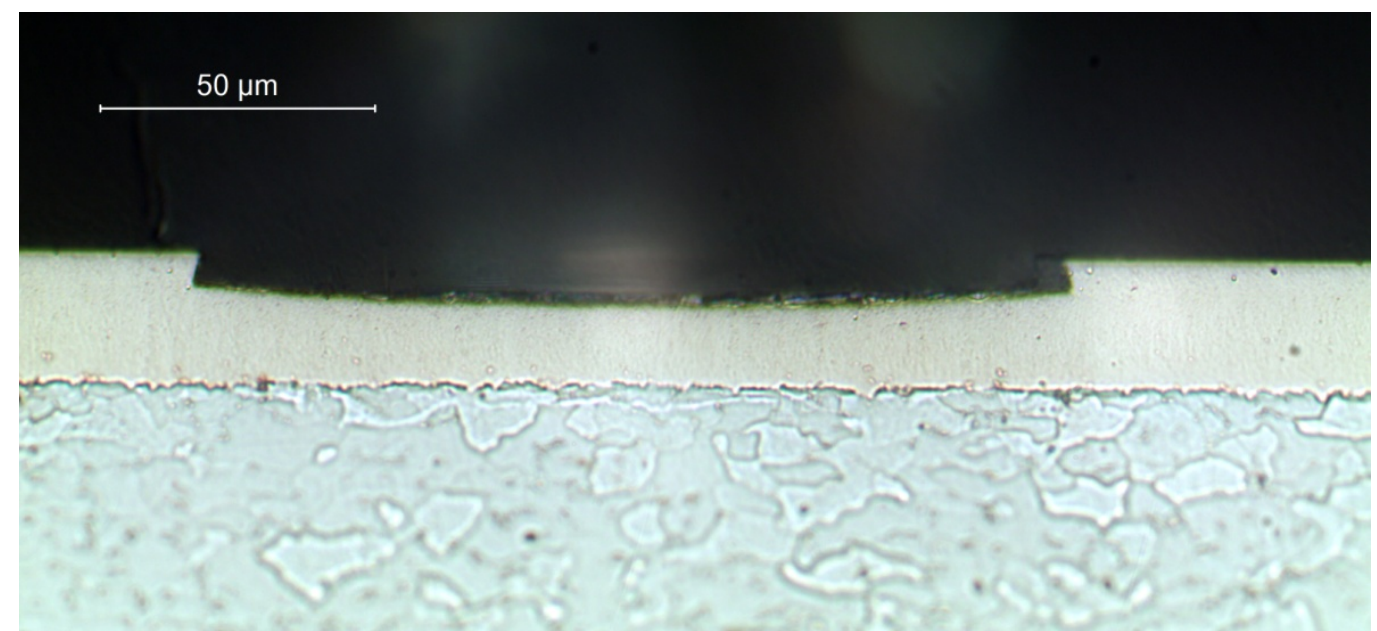

Figure 3 Cross-section of the profile of a circular defect in the nickel plate on a steel substrate.

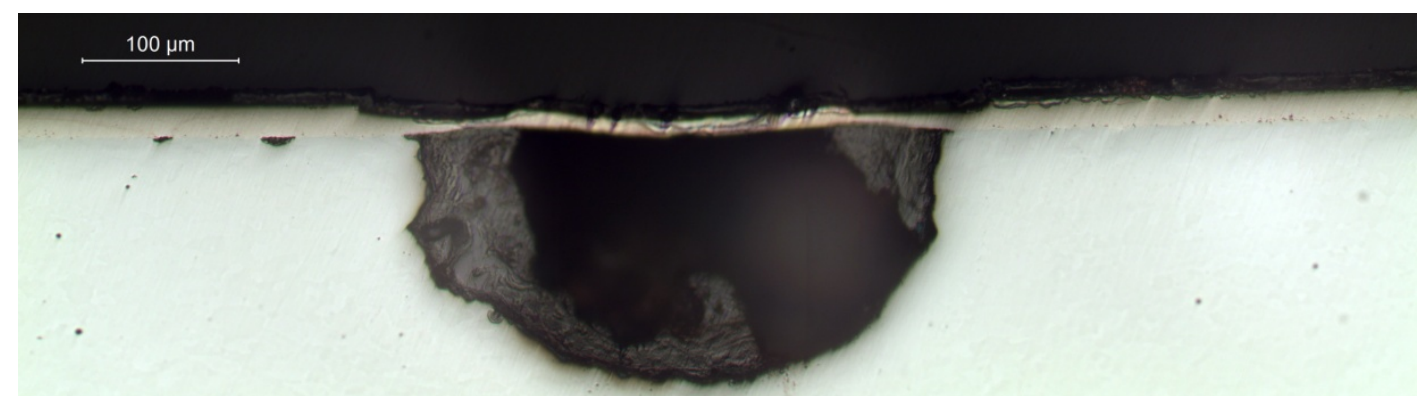

Figure 4 Cross-section through a circular defect with a corrosion pit beneath. 